\title{
ON THE LEIBNIZ RULE FOR RANDOM VARIABLES
}

\author{
ZOLTÁN LÉKA
}

Abstract. We prove a Leibniz-type inequality for the spread of (real-valued) random variables in terms of their $L^{p}$-norms. The result is motivated by the Kato-Ponce inequality and Rieffel's Leibniz property.

Mathematics subject classification (2010): Primary 46N30, 60E15, Secondary 26A51, 60A99. variable.

Keywords and phrases: Kato-Ponce inequality, Leibniz inequality, Laplacian, majorization, random

\section{REFERENCES}

[1] R. B. Bapat And T. E. S. Raghavan, Nonnegative Matrices and Applications, Cambridge University Press, Cambridge, 1997.

[2] Á. BeSENYei AND Z. LÉKA, Leibniz seminorms in probability spaces, J. Math. Anal. Appl., 429 (2015), 1178-1189.

[3] F. Bernicot, D. Maldonado, K. Moen and V. Naibo, Bilinear Sobolev-Poincaré inequalities and Leibniz-type rules, J. Geom. Anal., 24 (2014), 1144-1180.

[4] R. Bhatia, Matrix analysis, Springer-Verlag New York, 1997.

[5] R. Bhatia And T. SAno, Loewner matrices and operator convexity, Math. Ann., 344 (2009), $703-$ 716.

[6] D. Bertsimas, D. PaChamanova AND M. Sim, Robust linear optimization under general norms, Operations Research Letters, 32 (2004), 510-516.

[7] N. Bouleau And F. Hirsch, Dirichlet Forms and Analysis on Wiener space, de Gruyter Studies in Mathematics, De Gruyter, Berlin, 1991.

[8] A.-P. Calderón, Spaces between $L^{1}$ and $L^{\infty}$ and the theorem of Marcinkiewicz, Stud. Math., 26 (1966), 273-299.

[9] S. Chang And C. K. Li, Certain isometries on $\mathbb{R}^{n}$, Lin. Alg. Appl., 165 (1992), 251-265.

[10] F. Christ AND M. Weinstein, Dispersion of small-amplitude solutions of the generalized Korteweg-de Vries equation, J. Funct. Anal., 100 (1991), 87-109.

[11] M. Fukushima, Dirichlet Forms and Markov Processes, North Holland Mathematical Library, 1980.

[12] L. Grafakos, Modern Fourier Analysis, Graduate Texts in Mathematics, Springer, New York, 2014.

[13] L. Grafakos And S. OH, The Kato-Ponce inequality, Comm. Partial Differential Equations, 39 (2014), 1128-1157.

[14] R. A. Horn, C. R. Johnson, Topics in Matrix Analysis, Cambridge University Press, Cambridge, 1991.

[15] T. Kato And G. Ponce, Commutator estimates and the Euler and Navier-Stokes equations, Comm. Pure Appl. Math., 41 (1988), 891-907.

[16] C. Kenig, G. Ponce, AND L. Vega, Well-posedness and scattering results for the generalized Korteweg-de Vries equation via the contraction principle, Comm. Pure Appl. Math., 46 (1993), $527-$ 620.

[17] J. Kigami, Analysis on Fractals, Cambridge University Press, Cambridge, 2001.

[18] F. Latrémolière, The dual Gromov-Hausdorff propinquity, J. Math. Pures Appl., 103 (2015), $303-$ 351.

[19] F. Latrémolière, The quantum Gromov-Hausdorff propinquity, Trans. Amer. Math. Soc., 368 (2016), 365-411. 
[20] F. LATrÉmolière, Quantum metric spaces and the Gromov-Hausdorff propinquity, preprint, http://arxiv.org/pdf/1506.04341.

[21] Z. LÉKA, Symmetric seminorms and the Leibniz property, J. Math. Anal. Appl., 452 (2017), 708-725.

[22] B. S. Mityagin, An interpolation theorem for modular spaces (Russian), Mat. Sb. (N. S.), 66 (1965), 473-482.

[23] C. Muscalu And W. Schlag, Classical and Multilinear Harmonic Analysis, vol. 2., Cambridge University Press, 2013.

[24] C. Muscalu, J. Pipher, T. Tao And C. Thiele, Bi-parameter paraproducts, Acta Math., 193 (2004), 269-296.

[25] J. Von Neumann, Some matrix inequalities and metrization of matrix-space, Rev. Tomsk Univ., 1 (1937), 286-300.

[26] G. K. Pedersen, Analysis Now, Springer-Verlag, 1989.

[27] M. A. RiefFel, Metrics on state spaces, Doc. Math., 4 (1999), 559-600.

[28] M. A. RIEFFEL, Leibniz seminorms for "matrix algebras converge to the sphere", Quanta of Maths 11, Amer. Math. Soc., Providence, RI, 2010, 543-578.

[29] M. A. RIEFFEL, Non-commutative resistance networks, SIGMA Symmetry Integrability Geom. Methods Appl., 10 (2014), 2259-2274.

[30] M. A. RiefFel, Standard deviation is a strongly Leibniz seminorm, New York J. Math., 20 (2014), $35-56$.

[31] B. Simon, Convexity: An Analytic Viewpoint, Cambridge University Press, 2011.

[32] N. Weaver, Lipschitz algebras and derivations of von Neumann algebras, J. Funct. Anal., 139 (1996), 261-300.

[33] N. Weaver, Lipschitz algebras, World Scientific, 1999.

[34] B. Wu, C. Ding, D. Sun AND K.-C. Toh, On the Moreau-Yosida regularization of the vector $k$-norm related functions, SIAM Journal on Optimization, 24 (2014), 766-794. 